\title{
Correction to: Rule Injection-Based Generative Adversarial Imitation Learning for Knowledge Graph Reasoning
}

Sheng Wang, Xiaoying Chen, and Shengwu Xiong

\section{Correction to:}

Chapter "Rule Injection-Based Generative Adversarial

Imitation Learning for Knowledge Graph Reasoning" in: K. Karlapalem et al. (Eds.): Advances in Knowledge Discovery and Data Mining, LNAI 12714, https://doi.org/10.1007/978-3-030-75768-7_27

In the originally published version of chapter 27, the name of the author Xiaoying Chen was spelled incorrectly. This has been corrected. 